\title{
A comparison of surgical outcomes between endoscopic and robotically assisted thyroidectomy: the authors' initial experience
}

\author{
Brian Hung-Hin Lang $\cdot$ Man-Po Chow
}

Received: 16 June 2010/ Accepted: 1 November 2010/Published online: 19 November 2010

(C) The Author(s) 2010. This article is published with open access at Springerlink.com

\begin{abstract}
Background The gasless, transaxillary endoscopic thyroidectomy (GTET) offers a distinct advantage over the conventional open operation by leaving no visible neck scar, and in an attempt to improve its ergonomics and surgical outcomes, the robotically assisted thyroidectomy (RAT) was introduced. The RAT uses the same endoscopic route as the GTET but with the assistance of the da Vinci S robotic system. Excellent results for RAT have been reported, but it remains unclear whether RAT offers any potential benefits over GTET.

Methods From June to December 2009, 46 patients underwent endoscopic thyroidectomy. Of these patients, 39 had surgery without the robot (GTET) and 7 had surgery with the robot (RAT). Demographics, surgical indications, operative findings, and postoperative outcomes were compared between the two groups. All the patients were followed up for at least 6 months after surgery.

Results Patient demographics, surgical indications, and extent of resection were similar between the two groups. The median total procedure time was significantly longer for RAT (149 $\mathrm{min}$ ) than for GTET (100 $\mathrm{min} ; p=0.018$ ), but the contralateral recurrent laryngeal nerve was more likely to identified in RAT (100\%) than in GTET (42.9\%; $p=0.070)$. On the average, GTET needed one more surgical assistant than RAT ( 1 vs. $0 ; p<0.001)$. The median
\end{abstract}

B. H.-H. Lang - M.-P. Chow

Department of Surgery, The University of Hong Kong,

Hong Kong SAR, China

B. H.-H. Lang ( $\square)$

Division of Endocrine Surgery, Department of Surgery,

Queen Mary Hospital, 102 Pokfulam Road,

Hong Kong SAR, China

e-mail: blang@hkucc.hku.hk pain score on postoperative day 0 was significantly higher with RAT than with GTET (4 vs. $2 ; p=0.025$ ) but was similar on day 1 . Blood loss, hospital stay, and surgical complications were similar in the two groups.

Conclusions In our early experience, adding the da Vinci $S$ robot to GTET increased the total procedure time and resulted in a higher pain score on day 0 but eliminated the need for any surgical assistant at the time of the operation.

Keywords Endoscopic thyroidectomy $\cdot$ Robotic thyroidectomy · Robotically assisted thyroidectomy · Surgical outcomes - Surgical technique · Thyroid surgery

Since the first report of endoscopic parathyroidectomy in 1996, various endoscopic thyroid techniques or approaches have been described [1]. In general, these techniques can be broadly categorized based on the site of the incision relative to the neck [2]. Incisions made in the neck area are classified as the direct or cervical approach, whereas incisions made away from the neck such as in the chest or axillary area are classified as the indirect or extracervical approach [3]. Although the latter approach requires more tissue dissection, it offers superior cosmesis because there is no visible neck scar, and in experienced hands, similar surgical outcomes to open thyroidectomy could be achieved [4]. For this reason, it has been widely adopted in some Asian countries [2].

The gasless, transaxillary endoscopic thyroidectomy (GTET) is a well-established extracervical approach [5]. Compared with other approaches, especially those that use gas insufflations, GTET offers an excellent magnified endoscopic view while posing little or no risk of hypercapnia, air embolism, or subcutaneous emphysema [6]. However, GTET remains technically challenging because 
of the small working space and the limitations of current endoscopic instruments [5, 7, 8].

In 2007, a Korean group aiming to improve the ergonomics of GTET pioneered the gasless, transaxillary robotically assisted thyroidectomy (RAT), which uses the same approach as GTET but with the assistance of a robot. Their initial experience was subsequently reported in 2009, and their techniques were reproduced at two centers in the United States [9-12].

Despite the higher cost and bulkiness of the robot, it offers advantages such as improved motion of the endoscopic instruments (the so-called "endowrists"), availability of a three-dimensional view, and ability to damp the physiologic tremors. However, questions remain as to whether the addition of the robot will lead to better surgical outcomes.

To our knowledge, no direct comparison between GTET and RAT has been reported. This report serves as an initial comparison of surgical outcomes between the two procedures.

\section{Patients and methods}

From June 2009 to December 2009, 186 patients underwent thyroid resection. Of these patients, 46 had the endoscopic approach, 39 without robot assistance (GTET) and 7 with robot assistance (RAT). The inclusion criteria for the endoscopic approach specified age younger than 60 years as well as a dominant nodule smaller than $4 \mathrm{~cm}$ in benign cases and smaller than $2 \mathrm{~cm}$ in potentially malignant cases.

Before surgery, all the patients who met the inclusion criteria were given a choice of the open or the endoscopic approach. Those choosing the latter were given a further option of either GTET or RAT. Before surgery, all the patients underwent an ultrasonic examination to determine the location and size of the dominant nodule. Fine-needle aspiration cytology (FNAC) was performed for nodules larger than $1 \mathrm{~cm}$ or nodules with suspicious malignant features [13].

The RAT technique was performed using the da Vinci S surgical robotic system (Intuitive Surgical, Sunnyvale, CA, USA). All the patients were subjected to surgery and managed by one surgical team. The median age of the cohort was 43.5 years (range, 19-57 years), and 45 $(97.8 \%)$ of the patients were women. The median size of the largest nodule was $2.5 \mathrm{~cm}$ (range, $0.5-3.5 \mathrm{~cm}$ ). All the patients had at least a 6-month follow-up period after surgery.

\section{Surgical technique}

Details on the techniques of GTET and RAT had been described previously $[6,10]$. The initial part of the two procedures was similar. Patients were positioned supine with one arm extended over the shoulder to shorten the distance between the incision and the neck. A small pillow was placed behind the neck area for some neck extension.

After prepping and draping, a 4- to 5-cm skin incision was made in the axilla, and a subcutaneous flap was raised over the anterior surface of the pectoralis major muscle and the clavicle under direct vision. For bilateral resection, the side with the dominant nodule or a suspicious fine-needle aspiration (FNA) was generally the side of the axillary incision.

After exposure of the sternocleidomastoid muscle, the two arms of this muscle (i.e., the sternal and clavicular parts) were separated. The strap muscle was lifted from the thyroid capsule. An external retractor then was inserted through the axillary wound and lifted upward to maintain a working space over the thyroid gland.

For GTET, an additional 5-mm skin incision was made on the medial side of the chest about $2 \mathrm{~cm}$ below the lower horizontal line of the lower end of the axillary skin incision. A $30^{\circ} 10-\mathrm{mm}$ video camera and one working instrument were inserted through the axillary wound, and one additional instrument was inserted through the 5-mm chest port.

During thyroid dissection, the upper pole was retracted downward. Branches of the superior thyroid vessels were identified and individually divided using the Sonosurg (Olympus, Japan). Dissection of the upper pole was kept close to the capsule to avoid inadvertent injury to the external branch of the superior laryngeal nerve. The lower pole was dissected from the adipose tissue, and the inferior thyroid vein was divided close the thyroid gland. The ipsilateral lobe then was retracted medially, and the perithyroidal tissue was carefully dissected.

With careful dissection, the recurrent laryngeal nerve (RLN) was encountered and identified. For the contralateral side, the RLN was identified by anterolateral retraction of the lobe away from the trachea. After the first 15 cases, a nerve stimulator (Neurosign 100 machine; Magstim Clarify Company, Whitland, UK) was used to confirm the RLN function (Fig. 1). For RAT, instead of the 5-mm chest part, an 8-mm skin incision was made on the medial side of the anterior chest wall for insertion of the fourth robotic arm. The other three arms (for 1 camera and 2 working arms) were inserted through the axillary wound.

The actual steps of thyroid dissection were similar to those for GTET. As with GTET, the ipsilateral RLN was encountered and identified from the lateral side (Fig. 2) and the contralateral RLN from the medial side (Fig. 3). The resected specimen was retrieved through the axillary wound.

After hemostasis, a 3-mm closed suction drain was inserted through the main axillary wound. The skin was 


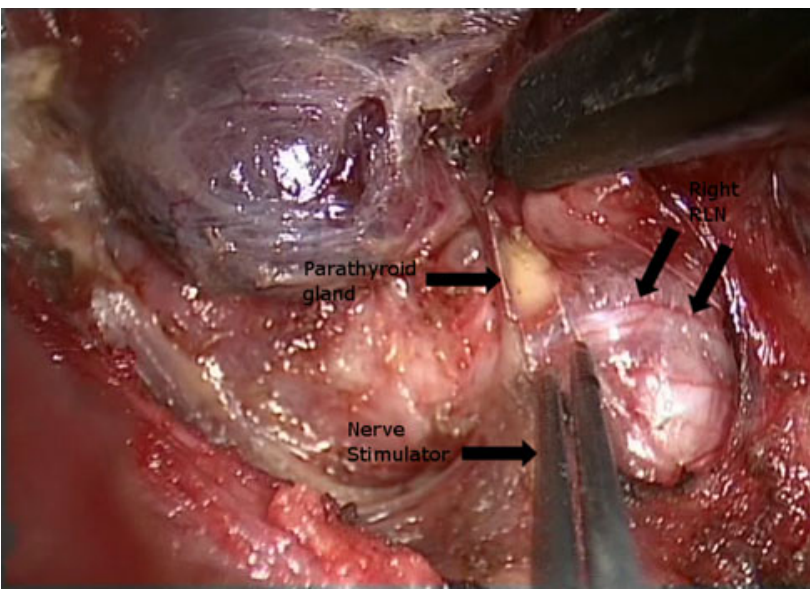

Fig. 1 Operative photo during endoscopic thyroidectomy showing the right lobe retracted upward and the ipsilateral (right) recurrent laryngeal nerve (RLN) running under it. A nerve stimulator probe was used to confirm the function of the RLN

closed subcuticularly. The same amount of oral analgesic was prescribed for each endoscopic procedure.

\section{Outcomes measured}

For each case, the weight of the excised specimen, the position and number of parathyroid glands identified, and the visualization of the RLN and ESBLN were recorded. The total procedure time was calculated from the time of skin incision to closure. For RAT, docking time was defined as the time taken for correct alignment of all four robotic arms into the thyroid area, and console time was defined simply as the time spent at the robot console by the operating surgeon. The number of surgical assistants required (not including the scrub nurse) also was recorded for each endoscopic procedure. A pain score using a scale of $0-10$ was obtained on days 0 and 1 after surgery.

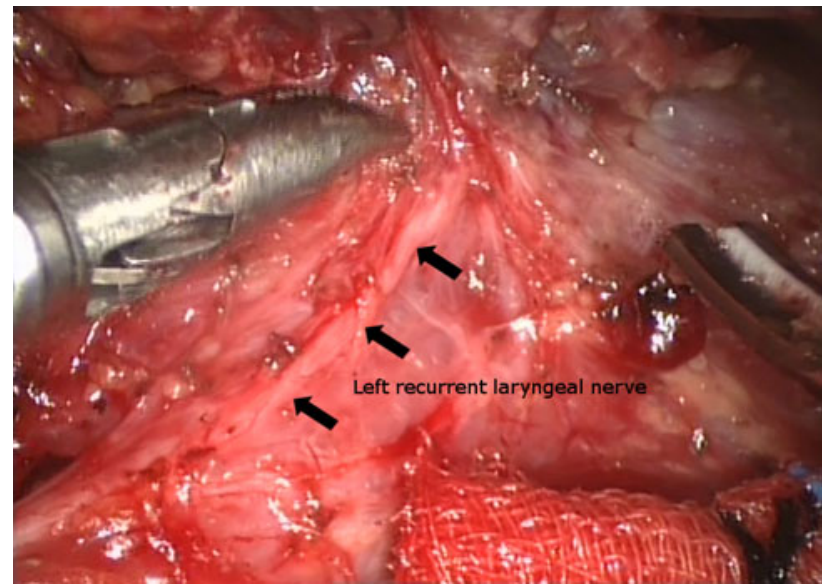

Fig. 2 Operative photo during robotically assisted thyroidectomy showing the course of the ipsilateral (left) recurrent laryngeal nerve

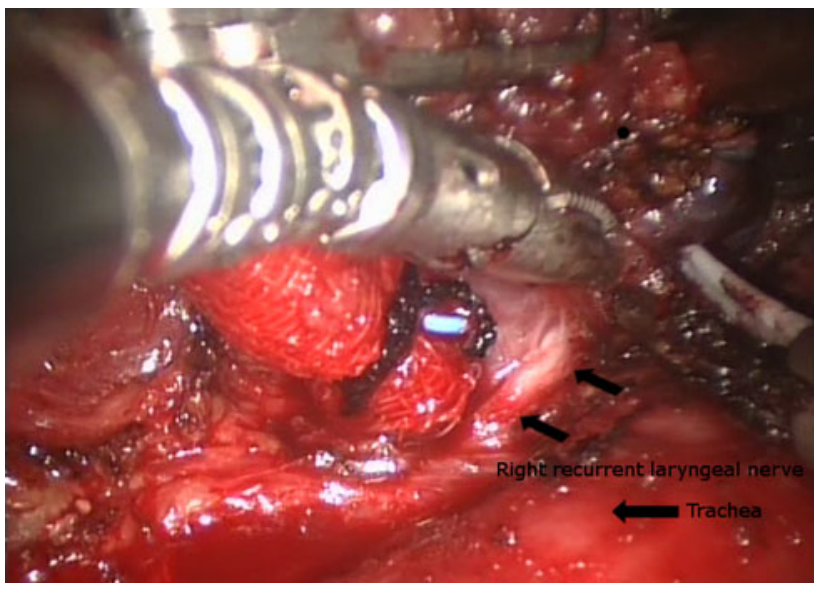

Fig. 3 Operative photo during robotically assisted thyroidectomy showing the course of the contralateral (right) recurrent laryngeal nerve with the lobe being retracted anterolaterally away from the trachea

In terms of postoperative management, for bilateral thyroid resection, serum calcium and phosphate were measured within $6 \mathrm{~h}$ and then every $12 \mathrm{~h}$ until the patient was stable. Calcium supplements \pm a vitamin $D$ analog were prescribed for symptomatic hypocalcemia or if the adjusted calcium level was less than $2 \mathrm{mmol} / \mathrm{l}$. Routine direct laryngoscopy was performed before surgery and within 1 week afterward to assess vocal cord function. Vocal cord palsy, documented by direct laryngoscopy, lasting more than 6 months after surgery was regarded as "permanent."

To calculate transient and permanent RLN injury rates, the number of nerves at risk was used as the denominator. Demographics, surgical indications, operative findings, and surgical outcomes were compared between the GTET and the RAT.

\section{Statistical analysis}

Statistical analysis was performed using the SPSS software package (version 18.0; SPSS, Inc., Chicago, IL, USA). Chisquare tests and Fisher's exact tests were used to compare dichotomous variables and the Mann-Whitney $U$ test to compare continuous variables between the two groups. A $p$ value less than 0.05 was considered statistically significant.

\section{Results}

Table 1 shows a comparison of demographics, surgical indications, extent of resection, size of the dominant nodule, and the final pathology between GTET and RAT. Age at operation, gender, surgical indications, tumor size, and 
final pathology were similar between the two groups. The current series included predominantly women (97.8\%), with only one man in the GTET group. The 15 patients $(32.6 \%)$ whose surgical indication was patient preference underwent benign FNAC and had no pressure symptoms. Indeterminate FNAC was the most common surgical indication in the two groups.

One patient in the RAT group had malignant FNAC, and the tumor size was $11 \mathrm{~mm}$, as determined by ultrasound. She ended up undergoing an ipsilateral central neck dissection at the time of thyroid resection, and two of six excised lymph nodes showed evidence of papillary thyroid carcinoma metastasis (pN1a). The woman subsequently received a dose of radioiodine ablation.

One patient in the GTET group underwent concomitant endoscopic excision of a parathyroid adenoma because of asymptomatic primary hyperparathyroidism. The parathyroid adenoma was located on the side of the excised lobe.

Although not statistically significant, a greater proportion of RAT patients $(66.7 \%)$ than GTET patients $(23.1 \%$, $p=0.176$ ) tended to undergo bilateral thyroid resection. The excised thyroid gland weighed significantly less in the RAT group than in the GTET group $(p=0.021)$. The median size of the dominant nodule tended to be smaller in the RAT group ( 2.5 vs. $1.6 \mathrm{~cm} ; p=0.244$ ), but the difference was not statistically significant.
Table 2 shows a comparison of operative findings between the GTET and RAT groups. The median number of parathyroid glands identified for both procedures were comparable. Identification of ipsilateral RLN with both procedures was more than $85 \%$, and there was no difference between the two groups. The chance of encountering and identifying the contralateral RLN tended to be higher in the RAT group (100 vs. 42.9\%), although this difference was not statistically significant $(p=0.070)$.

There was a significant difference in the median number of surgical assistants required because no surgical assistant was required at the time of thyroid resection. In all seven RAT cases, no surgical assistant was needed, whereas the median number of surgical assistants required in the GTET cases was one. However, the median total procedure time was significantly shorter in the endoscopic group (100 min) than in the robotic group (149 $\min ; p=0.018)$. When the procedures were stratified into uni- and bilateral resections, the GTET group still showed a tendency for a shorter total procedure. The median docking time was $15 \mathrm{~min}$, and the console time was $80 \mathrm{~min}$.

The blood loss and the open conversion rate were similar between the two groups. One patient in the GTET group required conversion because of uncontrolled bleeding from the upper thyroid pedicle. This was the third case in our series.

Table 1 Comparison of demographics, surgical indications, extent of resection, size of the dominant nodule, weight of gland, and final pathology between endoscopic thyroidectomy and robotically assisted thyroidectomy

\begin{tabular}{lcc}
\hline Variable & $\begin{array}{l}\text { Endoscopic thyroidectomy } \\
(n=39) n(\%)\end{array}$ & $\begin{array}{l}\text { Robotically assisted } \\
\text { thyroidectomy }(n=7) n(\%)\end{array}$ \\
\hline Median age at presentation: years (range) & $44.4(20.3-58.3)$ & $43.4(20.2-54.7)$ \\
Gender & $38(97.4)$ & $7(100.0)$ \\
Female & $1(2.6)$ & $0(0.0)$ \\
Male & & $1(14.3)$ \\
Surgical indications & $7(17.9)$ & $2(28.6)$ \\
Pressure symptoms & $13(33.3)$ & $3(42.9)$ \\
Patient preference & $19(48.7)$ & $1(14.3)$ \\
Indeterminate FNAC & $0(0.0)$ & $1.6(0.5-3.0)$ \\
Malignancy & $2.5(0.8-3.5)$ & $3(33.3)$ \\
Size of largest nodule on ultrasound: cm (range) & & $4(66.7)$ \\
Extent of resection & $29(77.0)$ & 0.139 \\
Unilateral thyroid resection & $10(23.1)$ & $6(85.7)$ \\
Bilateral thyroid resection & & $0(0.0)$ \\
Final pathology & $29(74.4)$ & $0(0.0)$ \\
Nodular hyperplasia & $5(12.8)$ & $1(14.3)$ \\
Follicular adenoma & $1(2.6)$ & 0.176 \\
Grave's disease & $4(10.3)$ & 0.493 \\
Differentiated thyroid carcinoma & & \\
\hline
\end{tabular}

FNAC fine-needle aspiration cytology 
Table 2 Comparison of operative findings between endoscopic thyroidectomy and robotically assisted thyroidectomy ${ }^{\mathrm{a}}$

\begin{tabular}{|c|c|c|c|}
\hline Variable & $\begin{array}{l}\text { Endoscopic } \\
\text { thyroidectomy }(n=39)\end{array}$ & $\begin{array}{l}\text { Robotically assisted } \\
\text { thyroidectomy }(n=7)\end{array}$ & $p$ value \\
\hline Weight of excised thyroid gland (g) & $19(10.7-37.0)$ & $11.3(6.0-67.1)$ & 0.021 \\
\hline $\begin{array}{l}\text { No. of parathyroid glands identified in unilateral } \\
\text { thyroid resection }\end{array}$ & $2(0-2)$ & $2(1-2)$ & 0.764 \\
\hline $\begin{array}{l}\text { No. of parathyroid glands identified in bilateral } \\
\text { thyroid resection }\end{array}$ & $2(0-3)$ & $3(1-4)$ & 0.096 \\
\hline Ipsilateral RLNs identified & 35 (89.7) & $6(85.7)$ & 1.000 \\
\hline Contralateral RLNs identified $^{\mathrm{b}}$ & $3(42.9)$ & $4(100)$ & 0.070 \\
\hline Total procedure time (min) & $100(50-220)$ & $149(92-190)$ & 0.018 \\
\hline Time for first 7 cases (min) & $120(95-220)$ & $149(92-190)$ & 0.004 \\
\hline Unilateral thyroid resection time (min) & $96(50-220)$ & $102.5(92-113)$ & 0.778 \\
\hline Bilateral thyroid resection time (min) & $135(98-198)$ & $161(148-190)$ & 0.327 \\
\hline Docking time (min) & NA & $15(10-19)$ & - \\
\hline Console time (min) & NA & $80(60-115)$ & - \\
\hline No. of surgical assistants & $1(1-2)$ & 0 & $<0.001$ \\
\hline Open conversions & $1(2.6)$ & $0(0.0)$ & 1.000 \\
\hline Blood loss (ml) & $20(10-60)$ & $30(20-60)$ & 0.723 \\
\hline
\end{tabular}

$R L N$ recurrent laryngeal nerve, $N A$ not applicable

a Values are given as $n$ (range) or $n(\%)$

b Applicable only in bilateral thyroid resection

Table 3 shows a comparison of postoperative outcomes between GTET and RAT. The median hospital stay was similar in the two groups. The pain score on day 1 after surgery was significantly higher in the RAT group $(p=0.025)$. Three patients $(5.5 \%)$ in the GTET group had a temporary RLN injury. The vocal cords returned to normal mobility after 2,3 , and 4 months, respectively.

One patient $(9.1 \%)$ in the RAT group had permanent RLN injury because the vocal cord did not return to normal function after 7 months. In all four cases, the side of the injured RLN was clearly identified and confirmed by a nerve stimulator except the one with the permanent injury, for which no nerve stimulator signal was detected at the end of the procedure. No patient in either group experienced postoperative hypocalcemia.

\section{Discussion}

Since the first reported endoscopic procedure in 1996, a great number of endoscopic thyroidectomy techniques and approaches have been described in the literature, with some involving small incisions in the neck (the cervical approach) and others involving incisions away from the neck (the extracervical approach) [2,3]. The latter offers the distinct advantage of no visible neck scar but has been criticized for its "maximal invasiveness" due to the amount of flap dissection required in reaching the thyroid gland [14]. Nevertheless, this approach has been adopted in a number of Asian countries because a "scarless" neck is important.

The gasless transaxillary approach is a well-established extracervical approach. Besides the excellent cosmesis, it provides clear magnification of vital structures while allowing for a fume-free endoscopic view [6]. However, because of the small confined space and the limited movements of current endoscopic instruments, the procedure poses a technical challenge. To overcome this and improve the ergonomics, RAT was developed. The group who pioneered RAT recently found less neck discomfort and fewer swallowing symptoms with RAT than with open thyroidectomy [15], but questions remain as to whether the addition of a robot in GTET would lead to better outcomes.

Because these procedures were primarily aimed at improving the cosmesis, our series comprised predominantly women, with only one man in the GTET group and none in the RAT group. In terms of performing either GTET or RAT, the authors would not anticipate any noticeable difference in techniques between the genders.

It was interesting to note that patients preferred GTET over RAT by a ratio of almost 5.6-1. This preference existed although both GTET and RAT were explained as carrying similar benefits and risks and began in the same period. Perhaps, this preference was related to patients' perception that RAT was a newer procedure than GTET with possible higher risks. 
Table 3 Comparison of postoperative outcomes between endoscopic thyroidectomy and robotically assisted thyroidectomy ${ }^{\mathrm{a}}$

\begin{tabular}{llll}
\hline Variable & $\begin{array}{l}\text { Endoscopic thyroidectomy } \\
(n=39)\end{array}$ & $\begin{array}{l}\text { Robotically assisted } \\
\text { thyroidectomy }(n=7)\end{array}$ \\
\hline Hospital stay (days) & $2(1-4)$ & $2(1-3)$ & 0.841 \\
Pain score on day 0 & $2(1-5)$ & $4(2-4)$ & 0.025 \\
Pain score on day 1 & $2(1-5)$ & $2(1-4)$ & 0.530 \\
Surgical complications & & & $0(0.0)$ \\
Temporary RLN injuries $^{\mathrm{b}}$ & $3(5.5)$ & $1(9.1)$ & 0.152 \\
Permanent RLN injuries $^{\mathrm{b}}$ & $0(0.0)$ & $0(0.0)$ & - \\
Hypocalcemia & $0(0.0)$ & $0(0.0)$ & - \\
Hematoma formation & $0(0.0)$ & $0(0.0)$ & - \\
Infection $_{\text {Tracheal injury }}^{0(0.0)}$ & $0(0.0)$ & $0(0.0)$ & - \\
\hline
\end{tabular}

$R L N$ recurrent laryngeal nerve

a Values are given as $n$ (range) or $n(\%)$

b Percentages calculated by dividing by the total number of nerves at risk

Although RAT managed a relatively smaller dominant nodule and a lighter thyroid gland, the total procedure time was significantly longer than for GTET. Even when the total procedure time was stratified into uni- and bilateral thyroid resections, GTET still tended to have a shorter procedure time. To evaluate whether this was related to the difference in getting over the "learning curve" between the two procedures, the median procedure time for the first seven GTET cases was compared with that for the seven RAT cases, and the procedure time still was significantly longer for RAT.

Our finding appeared to concur with that of another group [16]. Because the initial steps of the two procedures were similar, the most plausible explanation for the longer procedure time was the extra time needed for docking the robot to the patient. In fact, the median docking time of 15 min was more than twice that reported in a larger series but similar to that in a smaller series [17, 18]. Perhaps, with increasing experience, our docking time would gradually decrease. Nevertheless, this finding somewhat highlights both the difficulty of docking the robot and the importance of team work in ensuring efficient robotic surgery.

Other operative findings such as the number of parathyroid glands identified and the percentage of ipsilateral RLN identified were similar between GTET and RAT. However, the identification rate for contralateral RLN tended to be higher with RAT. Our experience shows that availability of the third working arm is essential to achieving a higher rate of RLN identification.

In GTET, it was difficult to maneuver a third working instrument because it tended to collide with the camera and the other two working instruments. In contrast, with the flexibility of the robotic arms, the three instruments could be maneuvered easily in a small working space. In our opinion, the third robotic arm allowed for better countertraction between the trachea and the contralateral lobe during the medial dissection of the gland, enabling the RLN to be readily identified.

For the seven patients with unidentified contralateral RLN at the time of GTET, a small remnant $(<1 \mathrm{~g})$ had to be left behind at the Berry ligament to avoid inadvertent injury. However, our study could not show a significant difference in RLN injury due to a higher rate of RLN identification because the number of patients was relatively small.

It was a concern that three patients undergoing GTET did experience temporary ipsilateral RLN, and one patient undergoing RAT actually experienced a permanent ipsilateral RLN injury. The authors attributed these injuries to relative inexperience and postulated that these injuries were the result of either heat injury from the Sonosurg (in GTET)/Harmonic Scalpel (in RAT) or traction injury during dissection.

To facilitate the identification of RLN and to improve the safety of the endoscopic approach, the nerve stimulator was introduced and the safety principles for using the Sonosurg or Harmonic Scalpel such as keeping the hot active tip at least $2 \mathrm{~mm}$ away from RLN and using these instruments in short spurts were closely followed [6]. However, it should be noted that it is yet to be shown that routine use of the nerve stimulator or monitoring during thyroidectomy significantly reduces permanent RLN injury in experienced hands [19]. Our study did find a higher pain score on day 0 with RAT, and this could be related to the longer procedure time. However, the pain score became comparable on day 1. No other serious complications were observed.

It was interesting to note that the high cost has been one of the arguments against the routine use of the robot. 
Although this was true because the added cost of using the da Vinci S robot was approximately HK $\$ 10,000$ or US $\$ 1,300$ per procedure, our study did find that no surgical assistant was required at the time of RAT, whereas at least one assistant was required for GTET. The issue of whether having one less surgical assistant would lower the cost over the long term and therefore compensate for the higher initial cost of the robot probably is beyond the aim of this study. However, this would be an important factor to consider in future cost-benefit analyses comparing the two procedures.

To our knowledge, this was one of the first direct comparisons between the endoscopic approach with and without robot assistance. Because the authors did not acquire extensive experience with endoscopic procedures before the start of GTET and RAT, this report truly represents the early learning curve for both procedures and thus should provide valuable insights to surgeons contemplating a start in using these procedures.

Given the longer procedure time and the higher cost for RAT, a selective approach might seem more logical in the future. Because more complex procedures such as central neck and lateral neck dissections for differentiated thyroid carcinoma could be performed together with RAT, perhaps RAT could be reserved for bilateral thyroid resection and more complex procedures, with GTET reserved for unilateral resection [18].

\section{Conclusion}

In our early experience, adding the da Vinci $S$ robot to GTET increased the total procedure time and resulted in a higher pain score on day 0 . However, the contralateral RLN was more often identified, eliminating the need for any surgical assistant at the time of operation.

Disclosures Brian Hung-Hin Lang and Man-Po Chow have no conflicts of interest or financial ties to disclose.

Open Access This article is distributed under the terms of the Creative Commons Attribution Noncommercial License which permits any noncommercial use, distribution, and reproduction in any medium, provided the original author(s) and source are credited.

\section{References}

1. Gagner M (1996) Endoscopic subtotal parathyroidectomy in patients with primary hyperparathyroidism. Br J Surg 83:875

2. Lang BH (2010) Minimally invasive thyroid and parathyroid operations: surgical techniques and pearls. Adv Surg 44:185-198
3. Slotema ET, Sebag F, Henry JF (2008) What is the evidence for endoscopic thyroidectomy in the management of benign thyroid disease? World J Surg 32:1325-1332

4. Jeong JJ, Kang SW, Yun JS, Sung TY, Lee SC, Lee YS, Nam KH, Chang HS, Chung WY, Park CS (2009) Comparative study of endoscopic thyroidectomy versus conventional open thyroidectomy in papillary thyroid microcarcinoma (PTMC) patients. J Surg Oncol 100:477-480

5. Yoon JH, Park CH, Chung WY (2006) Gasless endoscopic thyroidectomy via an axillary approach: experience of 30 cases. Surg Laparosc Endosc Percutan Tech 16:226-231

6. Kang SW, Jeong JJ, Yun JS, Sung TY, Lee SC, Lee YS, Nam KH, Chang HS, Chung WY, Park CS (2009) Gasless endoscopic thyroidectomy using transaxillary approach: surgical outcome of 581 patients. Endocr J 56:361-369

7. Ikeda Y, Takami H, Sasaki Y, Kan S, Niimi M (2000) Endoscopic neck surgery by the axillary approach. J Am Coll Surg 191:336-340

8. Chantawibul S, Lokechcareonlarp S, Pokawatana C (2003) Total video endoscopic thyroidectomy by an axillary approach. J Laparoendosc Adv Surg Tech A 13:295-299

9. Kang SW, Jeong JJ, Yun JS, Sung TY, Lee SC, Lee YS, Nam KH, Chang HS, Chung WY, Park CS (2009) Robot-assisted endoscopic surgery for thyroid cancer: experience with the first 100 patients. Surg Endosc 23:2399-2406

10. Kang SW, Jeong JJ, Nam KH, Chang HS, Chung WY, Park CS (2009) Robot-assisted endoscopic thyroidectomy for thyroid malignancies using a gasless transaxillary approach. J Am Coll Surg 209:e1-e7

11. Lewis CM, Chung WY, Holsinger FC (2010) Fesaibility and surgical approach of transaxillary robotic thyroidectomy without $\mathrm{CO}_{2}$ insufflation. Head Neck 32:121-126

12. Berber E, Heiden K, Akyidiz H, Milas M, Mitchell J, Siperstein A (2010) Robotic transaxillary thyroidectomy: report of 2 cases and description of the technique. Surg Laparosc Endosc Percutan Tech 20:e60-e63

13. Moon WJ, Jung SL, Lee JH, Na DG, Baek JH, Lee YH, Kim J, Kim HS, Byun JS, Lee DH, Thyroid Study Group, Korean Society of Neuro- and Head and Neck Radiology (2008) Benign and malignant thyroid nodules: US differentiation-multicenter retrospective study. Radiology 247:762-770

14. Tan CT, Cheak WK, Delbridge L (2008) "Scarless" (in the neck) endoscopic thyroidectomy (SET): an evidence-based review of published techniques. World J Surg 32:1349-1357

15. Lee J, Nah KY, Kim RM, Ahn YH, Soh E-Y, Chung WY (2010) Differences in postoperative outcomes, function, and cosmesis: open versus robotic thyroidectomy. Surg Endosc. doi:10.1007/ s00464-010-1113-z

16. Miyano G, Lobe TE, Wright SK (2008) Bilateral transaxillary endoscopic total thyroidectomy. J Paediatr Surg 43:299-303

17. Lee KE, Rao J, Youn YK (2009) Endoscopic thyroidectomy with the da Vinci robot system using the bilateral axillary breast approach (BABA): our initial experience. Surg Laparosc Endosc Percutan Tech 19:e71-e75

18. Kang SW, Lee SC, Lee SH, Lee KY, Jeong JJ, Lee YS, Nam KH, Chang HS, Chung WY, Park CS (2009) Robotic thyroid surgery using a gasless, transaxillary approach and the da Vinci S system: the operative outcomes of 338 consecutive patients. Surgery 146:1048-1055

19. Lang BH, Lo CY (2010) Technological innovations in surgical approach for thyroid cancer. J Oncol. doi:10.1155/2010/490719 